Review

\title{
The role of secretory leukocyte proteinase inhibitor and elafin (elastase-specific inhibitor/skin-derived antileukoprotease) as alarm antiproteinases in inflammatory lung disease
} Jean-Michel Sallenave

\author{
Edinburgh Medical School, Edinburgh, Scotland, UK
}

Received: 18 July 2000

Revisions requested: 3 August 2000

Revisions received: 7 August 2000

Accepted: 7 August 2000

Published: 23 August 2000
Respir Res 2000, 1:87-92

(c) Current Science Ltd (Print ISSN 1465-9921; Online ISSN 1465-993X)

\begin{abstract}
Secretory leukocyte proteinase inhibitor and elafin are two low-molecular-mass elastase inhibitors that are mainly synthesized locally at mucosal sites. It is thought that their physicochemical properties allow them to efficiently inhibit target enzymes, such as neutrophil elastase, released into the interstitium. Historically, in the lung, these inhibitors were first purified from secretions of patients with chronic obstructive pulmonary disease and cystic fibrosis. This suggested that they might be important in controlling excessive neutrophil elastase release in these pathologies. They are upregulated by 'alarm signals' such as bacterial lipopolysaccharides, and cytokines such as interleukin-1 and tumor necrosis factor and have been shown to be active against Gram-positive and Gram-negative bacteria, so that they have joined the growing list of antimicrobial 'defensin-like' peptides produced by the lung. Their site of synthesis and presumed functions make them very attractive candidates as potential therapeutic agents under conditions in which the excessive release of elastase by neutrophils might be detrimental. Because of its natural tropism for the lung, the use of adenovirus-mediated gene transfer is extremely promising in such applications.
\end{abstract}

Keywords: adenovirus, elafin/elastase-specific inhibitor/skin-derived antileukoprotease, elastase, inflammation, secretory leukocyte proteinase inhibitor

\section{Introduction}

Cytokines form one of the major classes of chemical mediator responsible for initiating, regulating and terminating the inflammatory response. Their synthesis, switchon and switch-off mechanisms and their mode of action are tightly regulated in what is now classically called a cytokine network. Indeed, early cytokines, such as inter- leukin-1 (IL-1) and tumor necrosis factor (TNF), are synthesized very quickly, within $1 \mathrm{~h}$ of the onset of inflammation, or in response to stimuli such as bacterial lipopolysaccharides (LPS).

These cytokines set in motion the migration of inflammatory cells such as neutrophils and monocytes, whose function

$\mathrm{A} 1-\mathrm{Pi}=\alpha 1$-proteinase inhibitor; $\mathrm{ALP}=$ antileukoprotease; ARDS = acute respiratory distress syndrome; bp = base pair; COPD = chronic obstructive pulmonary disease; $\mathrm{ESI}=$ elastase-specific inhibitor; $\mathrm{IL}=$ interleukin; $\mathrm{kb}=$ kilobases; $\mathrm{LPS}=$ lipopolysaccharide; SKALP $=$ skin-derived antileukoprotease; SLPI = secretory leukocyte proteinase inhibitor; TNF = tumor necrosis factor. 
is to eliminate the injurious agent and restore homeostasis. To migrate from the vascular space and gain access to the inflammatory site through the interstitium, it has been hypothesized that these inflammatory cells use a variety of proteases (such as neutrophil and monocyte/macrophage metalloproteases and elastases).

To contain the potential injurious effects of excess release of these proteases, the host secretes large amounts of antiproteinases, which also seem to have developed in a parallel network, consisting of 'alarm' and 'systemic' inhibitors, the latter being synthesized principally in the liver [such as $\alpha 1$ proteinase inhibitor (A1-Pi) and antichymotrypsin].

We are particularly interested in the former group, which include the two low-molecular-mass proteinase inhibitors of the ALP family, antileukoprotease (ALP), also known as secretory leukocyte proteinase inhibitor [1], mucus proteinase inhibitor or bronchial inhibitor (it will henceforth be referred to as SLPI), and elastase-specific inhibitor (ESI), also known as elafin or skin-derived antileukoprotease (SKALP) $\left[^{\circ}\right]$. They are synthesized and secreted locally at the site of injury and are produced in response to primary cytokines such as IL-1 and TNF; they might therefore be part of a first wave of local, inducible defense in the antiproteinases network [3]. Although potentially secreted at many mucosal sites and sites of interface such as the skin $\left[2^{\circ}\right]$, this review addresses mainly the role of SLPI and elafin in the lung.

\section{SLPI}

\section{Gene structure, protein and antiproteinase activity}

SLPI is one of the two members of the ALP superfamily of proteinase inhibitors (the other being ESI/elafin; see below). The gene encoding SLPI [2.65 kilobases (kb) in length [1]] seems to be a relatively nonpolymorphic, stable gene that can be modulated at both the transcriptional and translational levels [1]. Transfection studies with fusion elements composed of fragments of up to $1.2 \mathrm{~kb}$ of the $5^{\prime}$ flanking region of the SLPI gene demonstrated a high promoter activity in a 131 base pair (bp) fragment $(-115$ to +16$)$ relative to the transcription start site. Interestingly, Kikuchi et al [4] have recently delineated within this region a proximal $41-b p$ region that confers lung specificity for SLPI expression. It is a $11.7 \mathrm{kDa}$ protein consisting of 107 amino acid residues [1] and comprising two domains. It contains 16 cysteine residues that form eight disulfide bridges [1]. SLPI has been shown to inhibit human neutrophil elastase, cathepsin G, trypsin, chymotrypsin and chymase. Its major target is thought to be the human neutrophil elastase in view of its high affinity and kinetic constants (inhibition constant in the nanomolar range and $k_{\text {ass }}$ in the micromolar range) [5].

SLPI has been purified from different sources, including parotid, cervical, seminal and lung secretions [1].

\section{Cell and tissue distribution}

In lung, SLPI is produced in vitro by tracheal, bronchial, bronchiolar and type II alveolar cells, and by monocytes, alveolar macrophages and neutrophils $[1,6]$. It has also been shown to be produced in vivo by tracheal serous glands and bronchiolar Clara cells, and to be closely associated with elastin fibers in the alveolar interstitium [1]. Its roles in inflammatory cells such as macrophages or neutrophils are uncertain but antibacterial or anti-inflammatory actions have been proposed (see below). Outside the lung, it is secreted in a variety of mucosal sites (leading to its alternative name, mucosal proteinase inhibitor [1]).

\section{ESI/elafin/SKALP}

\section{Gene structure, protein and antiproteinase activity}

The neutrophil elastase inhibitor ESI/elafin (the other member of the ALP superfamily of proteinase inhibitors; reviewed in $\left[2^{\circ}\right]$ ) was first identified as a 'non-SLPI' lowmolecular-mass anti-elastase by Hochstrasser et al [7] and Kramps and Klasen [8], and further characterized by us in bronchial secretions [9] and by Wiedow et al and Molhuizen et al in the skin [10,11]. The sequence of the gene for ESI [12] showed that it is approx. $2.3 \mathrm{~kb}$ long, and is composed of three exons and two introns and containing typical $5^{\prime}$ TATA and CAAT boxes, as well as $5^{\prime}$ regulatory sequences such as activator protein-1 and nuclear factor- $\kappa \mathrm{B}$ sites $\left[2^{\circ}\right]$. Zhang et al demonstrated that a positive regulatory cis-element present in the region between -505 and $-368 \mathrm{bp}$ is responsible for the upregulation of the elafin gene in normal breast epithelial cells (reviewed in $\left[2^{\circ}\right]$ ). Further characterization will be needed to determine whether this region is tissue-specific or is also important for expression in lung cells. The molecule is composed of 117 amino acid residues, including a hydrophobic signal peptide of 22 residues. Elafin is part of a 'four-disulfide' core protein family that has recently been termed the trappin family [ $\left.2^{\circ}\right]$. Elafin can be divided into two domains, the carboxy-terminal domain containing the antiproteinase active site and the amino-terminal domain containing characteristic VKGQ sequences (in singleletter codes for amino acids). These sequences allow the elafin molecule to glue itself into polymers and bind other interstitial molecules through transglutamination [2]. This feature could make elafin maximally effective as a tissuebound inhibitor as opposed to $\mathrm{A} 1-\mathrm{Pi}$, which is present in large amounts in the circulation. SLPI has also been suggested to have a locally protective role against neutrophilic damage, presumably because of its small size and negative charge. The elafin molecule shows a $40 \%$ homology with the SLPI molecule and the active sites of both inhibitors are very similar.

Like SLPI, elafin has a high content of cysteine residues [2 ${ }^{\circ}$, which are arranged in four disulfide bonds in the C-terminal proteinase-inhibiting region $\left[2^{\circ}\right]$; a partial crystal structure, not containing the VKGQ sequences (a major 
feature of the molecule) has recently been determined [2॰]. Elafin has been shown to be more specific in its spectrum of inhibition than SLPI: it inhibits pig pancreatic elastase, human neutrophil elastase and proteinase-3 [2].

\section{Cell and tissue distribution}

As mentioned above, elafin was first demonstrated in the skin and in lung secretions but is also present at mucosal sites in many tissues. Its purification from sputum, its presence in tracheal biopsies and bronchoalveolar lavage from both normal subjects and patients [13], and its synthesis by Clara cells and type II cells indicate, as for SLPI, a tracheo-bronchioalveolar origin. It is present in highest concentrations in sputum (at approximately 10\% of the concentration of SLPI). We have recently investigated its presence in the peripheral lung and have shown by immunohistochemistry that macrophages are a primary source (J-M Sallenave, unpublished data).

\section{Regulation of SLPI and elafin}

It has been shown that 'alarm signals' such as bacterial LPS, IL-1, TNF, neutrophil elastase and defensins are able to switch on the production of these inhibitors $[3,14,15]$; conversely, anti-inflammatory and 'remodelling' cytokines such as transforming growth factor- $\beta$ can switch them off (this has been demonstrated at least for SLPI [16]). By comparison, 'systemic inhibitors' such as $\mathrm{A} 1-\mathrm{Pi}$ and antichymotrypsin are upregulated mainly by a later wave of cytokines such as those of the IL-6 family (IL-6 and oncostatin M), suggesting that these different inhibitors might be physiologically involved at discrete and different time points as well as different locations. Interestingly, interferon- $\gamma$ was shown to inhibit the LPS-induced SLPI upregulation in macrophages, suggesting a regulation of SLPI at the interface between innate and adaptive immunity [17 $\left.{ }^{\bullet \bullet}\right]$.

\section{Role of SLPI and elafin in innate immunity}

In addition to their proteinase inhibitory properties, which were historically first identified [5,7-11], and given their biochemical characteristics (low-molecular-mass cationic peptides, heavily disulphide bonded, with tissue distribution at mucosal sites), it followed that SLPI and elafin were good candidates for 'defensin-like' molecules. Indeed, they have recently been shown to have antimicrobial properties in vitro against bacteria, fungi and, potentially, HIV $\left[2^{\bullet}, 18\right]$. In vivo, elafin is also active against Pseudomonas aeruginosa (see below). In addition to these direct effects on microbes, it has also recently been shown in vitro that LPS is able to upregulate SLPI production in macrophages [15] and that the addition of recombinant SLPI to human monocytes or the transfection of macrophages with SLPI or elafin downregulates pro-inflammatory mediators such as TNF and matrix metalloproteinases on stimulation with LPS, for example $[15,19]$. This suggests that these inhibitors might also function to interfere directly (by binding to LPS) or indirectly (by downregulat-
Figure 1

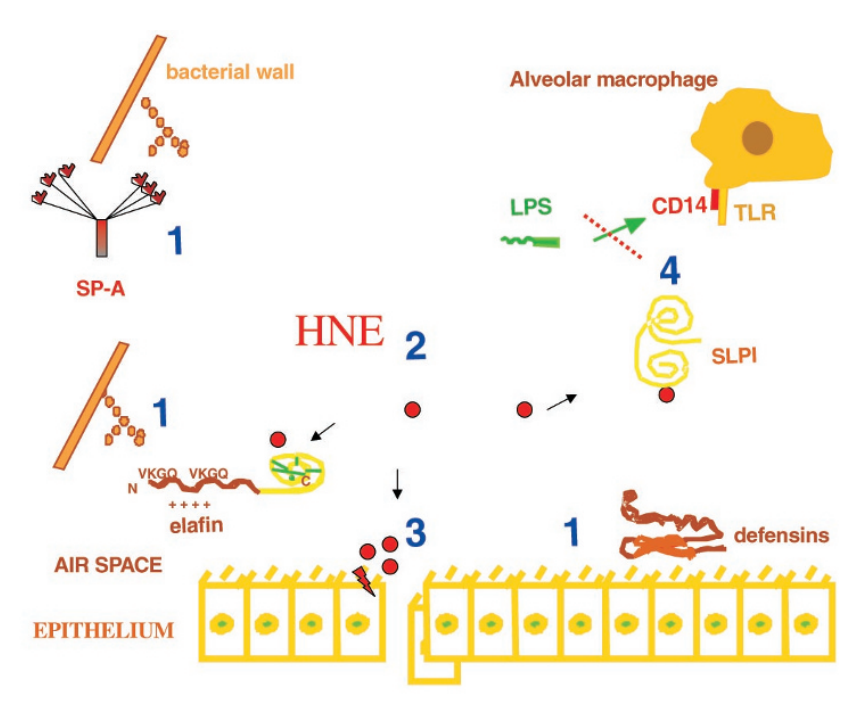

Role of SLPI and elafin in innate immunity. A representation of the alveolar space (delineated by the epithelium) and some of the molecules believed to be involved in innate immunity is shown. SLPI and elafin are elastase inhibitors that might be ideally placed to fight infection together with other molecules of the collectin family [such as the surfactant proteins A (SP-A)] and defensins (indicated by 1). In addition, SLPI and elafin have a role in modulating inflammation by inhibiting the neutrophil elastase (HNE, indicated by 2) and its potential deleterious effects on epithelial cells (indicated by 3 ) or by interfering with the agonistic effects of LPS on alveolar macrophages (indicated by 4 , dotted line).

ing nuclear factor- $\kappa B$ function, for example [20 $\left.{ }^{\circ}\right]$ ) with LPS in a feedback fashion (see Fig. 1). LPS and SLPI seem also to be coupled in vivo in that LPS responsiveness is modulated by SLPI; indeed, macrophages derived from a mouse line naturally resistant to LPS $(\mathrm{C} 3 \mathrm{H} / \mathrm{HeJ}$, recently found to have a mutation on the gene encoding Toll-4) consistently expressed high levels of SLPI, in contrast with $\mathrm{C} 3 \mathrm{H} / \mathrm{HeN}$, a strain sensitive to LPS.

\section{Role of SLPI and ESI/elafin in lung pathophysiology}

As mentioned above, SLPI and elafin were first isolated in the lung from sputum secretions from patients with chronic obstructive pulmonary disease (COPD) as the most abundant elastase inhibitors. It followed that one of their roles might be in controlling excess elastase release from the neutrophils present in these secretions.

\section{COPD/emphysema and cystic fibrosis}

In contrast to the $\mathrm{A} 1-\mathrm{Pi}$ deficiency in patients with the genetic form of emphysema, no polymorphisms have been reported so far for SLPI or elafin, although such studies are scarce. There is therefore no definite indication of whether a deficit in either SLPI or elafin could be responsible for the development of COPD in patients that are 
Figure 2

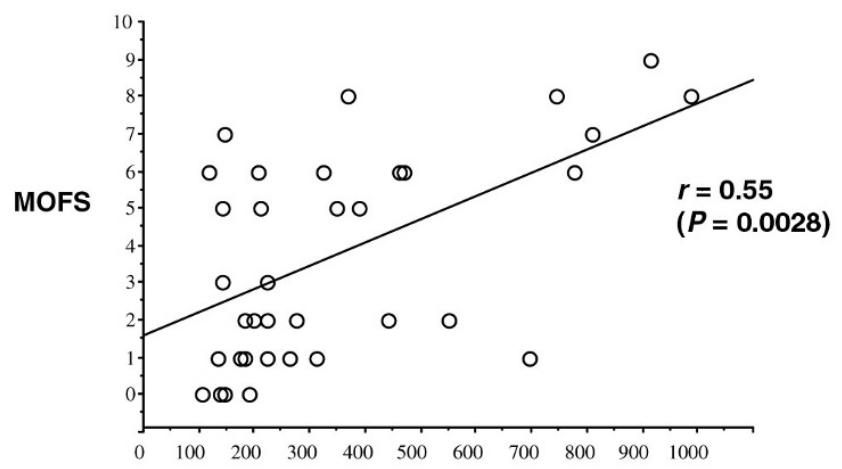

SLPI (nM)

SLPI and multiorgan failure score (MOFS) in patients with acute lung injury. The extent of organ involvement and severity of organ failure were quantified with a modified Goris organ failure score. SLPI levels were measured by enzyme-linked immunosorbent assay in bronchoalveolar lavage from 35 patients with acute lung injury. SLPI levels were correlated with MOFS $(r=0.55 ; P=0.0028)$. This suggests that SLPI could be used as a marker for acute lung injury. Indeed, we found recently that SLPI is increased in patients at risk of developing ARDS who later developed the disease, compared with patients at risk of ARDS who did not develop the disease [13].

otherwise A1-Pi sufficient. When the levels of SLPI were measured in relevant controls and patients with COPD, SLPI levels were found in significant amounts in bronchoalveolar lavage and sputum $[21,22]$. In contrast with the situation in genetic emphysema, in which A1-Pi levels are drastically decreased, we and others have found antigenic levels of SLPI to be increased in COPD and cystic fibrosis [6]. Compared with SLPI, elafin levels seem to be downregulated in these pathologies, suggesting that SLPI and elafin might be differently regulated in chronic situations.

Acute respiratory distress syndrome (ARDS) and pneumonia In ARDS, both SLPI and elafin are markedly increased in bronchoalveolar lavage fluid [13] compared with control subjects. Interestingly, SLPI BAL levels were positively correlated with the multiple organ failure score (MOFS, see Fig. 2), suggesting that antiproteases such as SLPI might be released in this pathology as an aborted and unsuccessful attempt to control injury. Furthermore, in patients with pneumonia, SLPI levels were found to be increased in serum [23], which is consistent with the hypothesis that these inhibitors are 'alarm inhibitors' whose role might be relevant at the onset of the inflammatory process.

\section{Asthma}

Although still a contentious issue, emerging evidence suggests that serine proteases might be important in the pathophysiology of asthma. Mast cell and leukocyte serine protease levels are elevated in the airways of asthmatic patients and one study has shown that patients with $\mathrm{A} 1-\mathrm{Pi}$ deficiency develop asthma more readily [24]. Although studies measuring SLPI and elafin in asthmatic patients are scarce, animal models of allergic diseases have shown SLPI to be of benefit in inhibiting both early and late phase events [25].

\section{Therapeutic potential of SLPI and elafin Use of recombinant proteins}

$\mathrm{A} 1-\mathrm{Pi}$ protein replacement therapy is actively being pursued in patients with severe $\mathrm{A} 1-\mathrm{Pi}$ deficiency and COPD. Although definite results from these studies are eagerly awaited, low-molecular-mass protease inhibitors such as SLPI and elafin have the advantages of size, diffusibility, and possible anchorage to the interstitium (see above), which is where the action is. Although no studies in patients have been reported with the use of recombinant elafin, the use of SLPI in normal subjects and in cystic fibrosis patients has been investigated. Unfortunately, these studies have been hampered by a short halflife and poor accessibility of the recombinant product to diseased areas in cystic fibrosis patients (reviewed in [1]).

\section{Gene therapy}

Although still in its infancy compared with pharmacological methods of drug delivery, gene therapy might be advantageous, owing to its less transient nature. Because of its natural tropism for the lung, adenovirus could be the vector of choice for gene therapy applications. Historically, most of these applications have been directed in the lung at chronic pathologies such as cystic fibrosis or cancer. This is by no means the best case, given the chronic nature of these pathologies in which a repeated administration of vectors would be necessary to achieve adequate levels of therapeutic transgenes. Unfortunately, the immune response directed against the currently available 'first and second generation' adenovirus vectors currently precludes their use in such settings. However, the generation of 'gutless' vectors (which express almost no viral genes) has shown to drastically improve the duration of transgene expression in rodent models [26*0. This type of vector is an important feature of adenovirus gene therapy techniques, but its generation remains technically challenging at present.

However, the constructs currently available are already useful for the study of experimental models in which immune responses are less of a concern and that mimic a clinical setting in which a therapeutic opportunity might exist between the presentation of patients and the development of irreversible acute inflammation. Such situations could include lung infections leading to pneumonia, ARDS or sepsis.

Indeed, success has been achieved in animal models of lung infections and endotoxemia in rodents, by using adenovirus vectors expressing molecules such as IL-12 
Table 1

\section{SLPI and elafin characteristics}

\begin{tabular}{|c|c|c|}
\hline Property & SLPI & ESI/elafin/SKALP \\
\hline Family name & Four disulfide core & Four disulfide core/trappin \\
\hline Molecular mass (kDa) & 11.7 & 9.8 \\
\hline Spectrum of protease inhibition & $\begin{array}{l}\text { HNE, trypsin, chymotrypsin, tryptase, } \\
\text { chymase, cathepsin G }\end{array}$ & HNE, pig pancreatic elastase, proteinase-3 \\
\hline Cell and tissue distribution in lung & $\begin{array}{l}\text { Tracheal, bronchial, Clara, alveolar type II cells, } \\
\text { monocytes, alveolar macrophages, neutrophils }\end{array}$ & $\begin{array}{l}\text { Tracheal, Clara, alveolar type II cells, } \\
\text { alveolar macrophages }\end{array}$ \\
\hline Regulatory stimuli & LPS, IL-1, TNF, HNE & IL-1, TNF, HNE \\
\hline Biological properties & $\begin{array}{l}\text { Antimicrobial, antiviral, anti-HNE, inhibition of } \\
\text { monocyte inflammatory potential }\end{array}$ & Antimicrobial, anti-HNE \\
\hline Gene therapy potential & Currently being explored & $\begin{array}{l}\text { Adenovirus-elafin protects against lung injury } \\
\text { (rodent model) }\end{array}$ \\
\hline
\end{tabular}

$\mathrm{HNE}=$ neutrophil elastase.

and TNF (lung infections) and IL-10 (endotoxemia) [27 $\left.-29^{\circ}\right]$. An important and useful feature of adenovirus administration is the compartmentalization of transgenes administered in the lung, which thereby prevents unwanted systemic effects of potentially toxic molecules such as TNF. Because of the previously mentioned characteristics of SLPI and elafin as 'alarm molecules' involved in the regulation of early events in the inflammatory process, the overexpression of these inhibitors would be of benefit in combating bacterial infections and their inflammatory sequelae. Our own studies have shown that the overexpression of elafin with an adenovirus vector (AdmCMV-elafin, in which CMV stands for cytomegalovirus) [30] significantly improved acute lung injury induced by Pseudomonas aeruginosa in C57/Bl6 mice (AJ Simpson and J-M Sallenave, unpublished data). The exact mechanism of the protection conferred by the elafin molecule is unclear at present and is still under investigation, but it might involve a dual mode of action, by killing the pathogens while dampening down the unwanted effects of neutrophil histotoxic molecules such as elastase.

\section{Conclusion}

SLPI and elafin/ESI/SKALP (see Table 1 for a summary) are gradually being recognized as potent locally produced elastase inhibitors whose characteristics allow them to be present first at the onset of inflammation. Strategies either to overexpress them (by using recombinant proteins or adenovirus-mediated delivery) or to inhibit them (by using neutralizing antibodies) have underlined their importance in vivo in the modulation of lung injury in asthma models [25], IgG immune-complex models [20*] or bacterial models, for example. Translating these findings into the clinical setting and identifying the best vector for administration and the cohort of patients most likely to benefit will remain, undoubtedly, a major focus of studies.

\section{Acknowledgements}

I acknowledge the support of the Salvesen Emphysema Research Trust, MRC-UK, the Wellcome Trust, the Scottish Hospital Endowment Research Trust, the British Lung Foundation, the Canadian Cystic Fibrosis Foundation and MRC-Canada. I thank the other members of my group, particularly $\mathrm{Dr}$ GA Cunningham, Dr PT Reid, Dr AJ Simpson, Mr ME Marsden and Professor C Haslett, for their involvement with the published work on SLPI and elafin, and also Professor J Gauldie, Dr SC Donnelly for his help in the study of acute lung injury, and Dr K Farmer for reviewing this manuscript.

\section{References}

Articles of particular interest have been highlighted as:

- of special interest

-. of outstanding interest

1. Stolk J, Hiemstra P: Recombinant SLPI: emphysema and asthma. In Emphysema and Infection. Edited by Stockley RA. Basel: Birkhauser; 1999:55-67. [Molecular Biology of the Lung, vol 1.]

2. Schalkwijk J, Wiedow $O$, Hirose $S$ : The trappin gene family: proteins - defined by an $\mathrm{N}$-terminal transglutaminase substrate domain and a C-terminal four-disulphide core. Biochem J 1999, 340:569-577.

This is an exhaustive review of the structure and functions of the members of the trappin family, to which elafin belongs.

3. Sallenave J-M, Schulmann J, Crosley J, Jordana M, Gauldie J: Regulation of secretory leukocyte proteinase inhibitor (SLPI) and elastase-specific inhibitor (ESI/elafin) in human airway epithelial cells by cytokines and neutrophilic enzymes. Am J Respir Cell Mol Biol 1994, 11:733-741.

4. Kikuchi T, Abe T, Satoh K, Narumi K, Sakai T, Abe S, Shindoh S, Matsushima $\mathrm{K}$, Nukiwa $\mathrm{T}$ : cis-acting region associated with lung-cell specific expression of the secretory leukoprotease inhibitor gene. Am J Respir Cell Mol Biol 1997, 17:361-367.

5. Boudier C, Bieth JG: The proteinase-mucus proteinase inhibitor binding stoichiometry. J Biol Chem 1992, 267:4370-4375.

6. Sallenave J-M, Si-Tahar M, Cox G, Chignard M, Gauldie J: Secretory leukocyte proteinase inhibitor is a major leukocyte elastase inhibitor in human neutrophils. J Leukoc Biol 1997, 61:695-702.

7. Hochstrasser K, Albrecht GJ, Schonberger G, Rasche B, Lempart K: An elastase-specific inhibitor from human bronchial mucus. Isolation and characterization. Hoppe-Seyler's Z Physiol Chem 1981, 362:1369-1375.

8. Kramps JA, Klasen EC: Characterization of a low molecular weight anti-elastase isolated from human bronchial secretion. Exp Lung Res 1985, 9:151-165. 
9. Sallenave JM, Ryle AP: Purification and characterization of elastase-specific inhibitor. Sequence homology with mucus proteinase inhibitor. Biol Chem Hoppe-Seyler 1991, 372:13-21.

10. Wiedow O, Schroder J, Gregory H, Young JA, Christophers E: Elafin: an elastase-specific inhibitor of human skin. Purification, characterization, and complete amino acid sequence. J Biol Chem 1990, 265:14791-14795.

11. Molhuizen HOF, Alkemade HAC, Zeeuwen PLJM, de Johgh GJ, Wieringa B, Schalkwijk J: SKALP/elafin: an elastase inhibitor from cultured human keratinocytes. Purification, cDNA sequence, and evidence for transglutaminase cross-linking. J Biol Chem 1993, 268:12028-12032.

12. Sallenave J-M, Silva A: Characterization and gene sequence of the precursor of elafin, an elastase-specific inhibitor in bronchial secretions. Am J Respir Cell Mol Biol 1993, 8:439-445.

13. Sallenave J-M, Donnelly SC, Grant IS, Robertson C, Gauldie J, Haslett C: Secretory leukocyte proteinase inhibitor is preferentially increased in patients with acute respiratory distress syndrome. Eur Respir J 1999, 13:1029-1036.

14. Van Wetering $S$, van der Linden AC, van Sterkenburg MA, de Boer WI, Kuijpers AL, Schalkwijk J, Hiemstra PS: Regulation of SLPI and elafin release from bronchial epithelial cells by neutrophil defensins. Am J Physiol Lung Cell Mol Physiol 2000, 278:L51-L58.

15. Jin FY, Nathan CF, Radzioch D, Ding A: Lipopolysaccharide-related stimuli induce expression of the secretory leukocyte protease inhibitor, a macrophage-derived lipopolysaccharide inhibitor. Infect Immun 1998, 66:2447-2452.

16. Jaumann F, Elssner A, Mazur G, Dobmann S, Vogelmeier C: Transforming growth factor-beta1 is a potent inhibitor of secretory leukoprotease inhibitor expression in a bronchial epithelial cell line. Eur Respir J 2000, 15: 1052-1057.

17. Jin FY, Nathan C, Radzioch D, Ding A: Secretory leukocyte protease - inhibitor: a macrophage product induced by and antagonistic to bacterial lipopolysaccharide. Cell 1997, 88:417-426.

This important article shows that SLPI can be considered as a molecule involved in the feedback regulation of LPS on macrophages. It also provides a potential link with the hyporesponsiveness of $\mathrm{C} 3 \mathrm{H} / \mathrm{HeJ}$ mice to LPS, by showing that macrophages derived from these mice overexpress SLPI.

18. Simpson AJ, Maxwell Al, Govan JRW, Haslett C, Sallenave JM: Elafin (elastase-specific inhibitor) has anti-microbial activity against Gram-positive and Gram-negative respiratory pathogens. FEBS Lett 1999, 452:309-313.

19. Zhang Y, DeWitt DL, McNeely TB, Wahl SM, Wahl LM: Secretory leukocyte protease inhibitor suppresses the production of monocyte prostaglandin $\mathrm{H}$ synthase-2, prostaglandin E2, and matrix metalloproteinases. J Clin Invest 1997, 199:894-900.

20. Lentsch AB, Jordan AJ, Czermak BJ, Diehl KM, Younkin EM, Sarma V,

- Ward PA: Inhibition of NF-kappaB activation and augmentation of Ikappabeta by secretory leukocyte protease inhibitor during lung inflammation. Am J Pathol 1999, 154:239-247.

The authors suggest a new mechanism by which overexpression of SLPI in

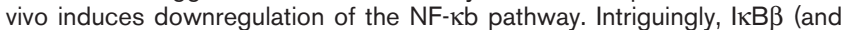
not $1 \kappa B \alpha$ ) seems upregulated by SLPI.

21. Morrison HM, Kramps JA, Burnett D, Stockley RA: Lung lavage fluid from patients with alpha-1 proteinase inhibitor deficiency or chronic obstructive bronchitis: anti-elastase function and cell profile. Clin Sci 1987, 72:373-381.

22. Stockley RA, Morrison HM, Smith S, Tetley T: Low molecular mass bronchial proteinase inhibitor and alpha-1 proteinase inhibitor in sputum and bronchoalveolar lavage. Hoppe-Seyler's Z. Physiol. Chem 1984, 365:587-595.

23. Asano S, Kida K, Koyama T, Wada H, Izawa Y, Hosoda K, Masuda K, Suzuki Y: A morphological study of lung secretory leukoprotease inhibitor in pneumonia. Am J Respir Crit Care Med 1995, 151: $1576-1581$.
24. Eden $E$, Mitchell D, Mehlman B, Khouli $H$, Nejat M, Grieco $M H$, Turino GM: Atopy, asthma, and emphysema in patients with severe alpha1 antitrypsin deficiency. Am J Respir Crit Care Med 1997, 156:68-74.

25. Wright CD, Havill AM, Middleton SC, Kashem MA, Lee PA, Dripps DJ, O'Riordan TG, Bevilacqua MP, Abraham WM: Secretory leukocyte protease inhibitor prevents allergen-induced pulmonary responses in animal models of asthma. J Pharmacol Exp Ther 1999, 289:10071014

26. Morral N, O'Neal W, Rice K, Leland M, Kaplan J, Piedra PA, Zhou H,

$\bullet \quad$ Parks RJ, Velji R, Aguilar-Cordova E, Wadsworth S, Graham FL, Kochanek S, Carey KD, Beaudet AL: Administration of helperdependent adenoviral vectors and sequential delivery of different vector serotype for long-term liver-directed gene transfer in baboons. Proc Natl Acad Sci USA 1999, 96:12816-12821.

This paper shows that helper-dependent adenoviral vectors (so called "gutted adenovirus") allowed for a very long period of gene expression (A1proteinase inhibitor ) in primates, with negligible toxicity to the liver. This has very interesting implications for human gene therapy in chronic settings, when the therapeutic agent should be present for extended periods.

27. Greenberger MJ, Kunkel SL, Strieter RM, Lukacs NW, Bramson J,

- Gauldie J, Graham FL, Hitt M, Danforth JM, Standiford TJ: IL-12 gene therapy protects mice in lethal Klebsiella pneumonia. J Immunol 1996, 157:3006-3012.

This report demonstrates that adenovirus vector is a powerful vector for expression of the Th1 cytokine IL-12, resulting in efficient clearance of Klebsiella pneumoniae.

28. Kolls J, Lei D, Nelson S, Summer WR, Greenberg S, Beutler B

- Adenovirus-mediated blockade of tumor necrosis factor in mice protects against endotoxic shock yet impairs pulmonary host defense. J Infect Dis 1995, 171:570-575.

Using an adenovirus coding for a protein capable of binding TNF, the authors confirm in this report that TNF can be either a 'friend' or a 'foe'. This report shows the possibility, using a gene therapy approach, of singling out TNF as a potential target in endotoxic shock.

29. Xing Z, Ohkawara Y, Jordana M, Graham FL, Gauldie J: Adenoviral - vector-mediated interleukin-10 expression in vivo: intramuscular gene transfer inhibits cytokine responses in endotoxemia. Gene Ther 1997, 4:140-149.

This report shows that the intramuscular expression of an IL-10 transgene resulted in a high concentration of it in blood. This concentration allowed for a marked therapeutic effect against endotoxemia and the resulting lung inflammation. This might have important implications for ARDS.

30. Sallenave J-M, Xing Z, Simpson AJ, Graham FL, Gauldie J: Adenovirus-mediated expression of an elastase-specific inhibitor (elafin): a comparison of different promoters. Gene Therapy 1998, 5:352-360

Author's affiliation: Rayne Laboratory, Centre for Inflammation Research, Edinburgh Medical School, Edinburgh, Scotland, UK

Correspondence: J-M Sallenave, Rayne Laboratory, Centre for Inflammation Research, Edinburgh Medical School, Teviot Place, Edinburgh EH8 9AG, Scotland, UK. Tel: +44 (0)131651 1324; fax: +44 (0)1316504384; e-mail: j.sallenave@ed.ac.uk 